\title{
Las prácticas de conciliación vida personal-vida laboral y su relación con el avance de la mujer en la jerarquía organizacional - El caso de las empresas grandes limeñas
}

\section{Resumen}

Las mujeres forman parte activa de nuestra economía. Sin embargo, los cargos que ocupan y las responsabilidades del hogar, entre otros factores, dan cuenta de que no existe igualdad de oportunidades laborales para hombres y mujeres. El ofrecimiento, por parte de las empresas, de prácticas de conciliación vida personal-vida laboral puede contribuir a que las mujeres desarrollen una línea de carrera. En este estudio se presenta un modelo que estima el porcentaje de mujeres que ocupa posiciones de nivel medio, en empresas grandes que operan en Lima, a través del número de prácticas de conciliación que ofrecen sus empresas.

\section{Palabras clave}

Flexibilidad laboral, equilibrio trabajo-vida, prácticas de conciliación. 


\section{Introducción}

Las mujeres se han incorporado a la fuerza laboral a nivel mundial con mucha fuerza. Sin embargo, aún no se puede hablar de igualdad de oportunidades laborales para hombres y mujeres. The Corporate Gender Gap Report (World Economic Forum 2010) muestra los resultados de encuestas aplicadas a los 100 más grandes empleadores de cada uno de los 30 países miembros de la OCDE (Organización para la Cooperación y el Desarrollo Económico) incluyendo a Brasil, Rusia, India y China. Según este informe, en promedio, en todos los países encuestados, las mujeres en la posición de CEO son poco menos que el $5 \%$. Esta cifra está altamente relacionada con lo que presenta el listado de empresas Fortune 500, pues aquí el porcentaje de mujeres CEO es sólo el $3 \%$ (Fortune 2010). En América Latina, un interesante artículo denominado Tierra de Hombres (García 2010), da cuenta de que en los consejos de administración de las 100 empresas más grandes de América Latina, sólo el $7 \%$ de 654 asientos disponibles es ocupado por mujeres. Como dato adicional, el autor comenta que muchas de estas mujeres pertenecen a las familias que controlan dichas empresas. DBM Perú realizó durante el año 2009 un estudio sobre diversidad en 68 empresas grandes en el Perú. En estas empresas, que tienen en promedio 523 colaboradores, sólo un $9 \%$ de mujeres ocupa puestos ejecutivos.

A nivel mundial, los hombres se encuentran empleados en una mayor proporción sobre su población total $(72.8 \%)$ si se compara con el de las mujeres (48\%). Un gran porcentaje de mujeres no participa en la actividad económica, lo cual da a suponer la existencia de un gran potencial humano no utilizado que favorecería al crecimiento económico (ILO 2010). En el Perú, la tasa de actividad masculina (PEA/PET, Población Económicamente Activa sobre Población en Edad de Trabajar) es de $82.9 \%$ y la tasa de actividad femenina es de $65.8 \%$ (Mintra 2009). Según la Encuesta Nacional de Uso del Tiempo 2010 (INEI 2011), las mujeres en edad de trabajar dedican 12 horas a la semana al cuidado de bebes, niños, niñas y adolescentes mientras que los hombres dedican 5 horas semanales a actividades similares. Estas cifras muestran que, aunque la presencia de la mujer en el mercado laboral es notoria, la igualdad de oportunidades para hombres y mujeres parece no estar presente, puesto que a la mujer se le dificulta alcanzar puestos de dirección en la jerarquía organizacional. Es por esta situación que surge el interés de explorar posibles medidas que contribuyan a que la mujer trabaje en igualdad de oportunidades.

\section{Objetivo del estudio}

Las prácticas de conciliación vida personal-vida laboral pueden contribuir a que hombres $y$ mujeres trabajen en igualdad de oportunidades $y$, en el caso de las mujeres, pueden ayudar a su desarrollo profesional (Barsh y Yee 2011). Debido a esto, se pretende reconocer si las prácticas de conciliación vida personal-vida laboral contribuyen a que las mujeres puedan desarrollar una línea de carrera en la empresa. La pregunta de investigación que se propone es si se puede estimar el porcentaje de mujeres que ocupa cargos de nivel medio a través de conocer el número de prácticas de conciliación vida personal-vida laboral que ofrece una empresa. Cabe mencionar que el estudio está limitado a empresas grandes que operan en la ciudad de Lima.

\section{Revisión de la literatura}

Los modelos tradicionalmente masculinos que existen en las empresas recompensan el permanecer largas jornadas en los centros laborales. Esto tenía sentido en los años cincuenta, cuando el $63 \%$ de las familias seguían este modelo tradicional en el cual el hombre era el sostén de la familia y la mujer se quedaba en casa (Benko y Weisberg 2007, en Brown 2010). Según Cabrera (2009, en Brown 2010) sólo el $17 \%$ de los hogares sigue hoy el modelo tradicional de los años cincuenta. Sin embargo, es la mujer la que aún tiene la responsabilidad de llevar la casa, los niños y su carrera. Según el Families and Work Institute (2002, en Brown 2010), dos de cada cinco hombres piensan que el lugar de una mujer es el hogar. Estas presiones 
pueden explicar la razón por la que mujeres de muy alto nivel profesional deciden dedicarse a una posición menos demandante luego de ser madres (Cabrera 2009, en Brown 2010).

Una encuesta de Catalyst sobre las mujeres que dejan su trabajo y luego vuelven a la fuerza laboral en Estados Unidos (Catalyst 2009) revela un dato interesante. Se cree que las mujeres dejan su trabajo fácilmente para quedarse en casa con sus hijos. Sin embargo, el estudio revela que las mujeres sienten grandes conflictos al dejar su trabajo y lo encuentran muy difícil. La razón es que han invertido mucho esfuerzo en su preparación profesional y el trabajo es realmente importante en sus vidas. Si dejan el trabajo no es por tener hijos sino porque los empleadores no les brindan las facilidades para combinar el trabajo con el resto de su vida.

Por otra parte, las diferencias en el sueldo existen entre hombres y mujeres sin importar que se tengan o no hijos. Un estudio de Catalyst (2009, en Brown 2010) indica que las mujeres con MBA —en todo el mundo- reportan estar detrás del hombre en nivel y salario desde su primer puesto de trabajo luego de obtener el MBA, independientemente de si tienen hijos. Algunas mujeres eligen no tener hijos, posponen el tenerlos o no gozan de sus licencias de maternidad para acortar la brecha. Las madres parecen estar expuestas a más discrepancias y menos oportunidades de promociones (Ranson 1998, en Brown 2010). Para Guillaume y Pochic (2007, en Brown 2010), las mujeres que posponen la maternidad se aseguran de que cuando tengan hijos tendrán posibilidades para contratar apoyo en casa, como nanas y personal de limpieza y así poder reducir su período de licencia por maternidad. Estas mujeres trabajan para adaptarse a las normas de carrera masculinas y no para obtener el balance trabajo-familia.

\section{El equilibrio trabajo-vida}

Edwards y Rothbard (2000) proponen que el trabajo y la familia son dos dominios de la vida humana fuertemente interconectados. La teoría de roles sugiere que los individuos experimentan conflicto entre roles cuando cumplir los requerimientos de un rol dificulta el cumplir con los requerimientos del otro rol. Así, el conflicto trabajo-familia es un tipo de conflicto entre roles (Powell y Greenhaus 2010).

El término balance trabajo-vida se utiliza actualmente pero ha derivado de los términos conflicto trabajo-familia e interferencia trabajofamilia. Por «trabajo» se entiende un empleo formal y pagado o trabajo que es desarrollado a cambio de un salario, y por «vida» se entiende todo aquello que se realiza fuera del ámbito laboral (Chang et al. 2010). De esta manera se enfatiza que todo individuo tiene la necesidad de ser apoyado para desarrollar una vida más allá del trabajo (Kossek et al. 2010). Frecuentemente, se utiliza el balance trabajo-familia o trabajo-vida como la ausencia de conflicto trabajo-familia o trabajo-vida (Grzywacz y Carlson 2007). Algunos autores proponen utilizar la categoría «labor recreacional» para referirse a momentos de ocio y de tiempo personal, como comer, dormir, entre otros (Ransome, 2007).

Para Chang et al. (2010) por balance se entiende el equilibrio entre los dominios del trabajo y de la vida. Si se utiliza la palabra conflicto o interferencia para referirse a la relación entre ambos dominios la aproximación es negativa. Utilizar las palabras balance o equilibrio tornan la definición más inclusiva (Chang et al. 2010). Un lugar de trabajo inclusivo será aquel en el que los individuos se sientan aceptados y valorados sin importar si son solteros o casados, si tienen hijos, si son heterosexuales o no, si trabajan medio tiempo o tiempo completo (Ryan y Kossek 2008). Los términos se han vuelto más inclusivos pero según Lewis et al. (2007) el término trabajo-vida no es adecuado porque implica que el trabajo $y$ el resto de la vida son mutuamente excluyentes. Lewis et al. (2007) sugieren el término «trabajopersona integrados a la vida».

El equilibrio trabajo-vida se define como el buen funcionamiento en aspectos laborales $y$ en aspectos personales con un mínimo conflicto de roles (Sturges y Guest 2004). La necesidad de alcanzar el equilibrio entre los ámbitos laboral y no laboral es una prioridad para muchas personas. Las consecuencias de un desequilibrio son enfermedades psicológicas, reducción en la satisfacción laboral y en la satisfacción con 
la vida y bajos niveles de compromiso (Sturges 2008). Sturges también alerta sobre el problema que puede acarrear el que los límites entre las actividades laborales y no laborales sean confusos. En ese caso, el cansancio y el estrés se transfieren del dominio laboral al no laboral. Las investigaciones han demostrado que cuando un individuo requiere de una política de equilibrio vida-trabajo y no la encuentra, se produce el efecto frustración (Ryan y Kossek 2008). La gente con altos niveles de conflicto tiende a estar menos satisfecha con su trabajo (Kossek y Ozeki 1998).

\section{Prácticas de conciliación trabajo-vida}

Kossek et al. (2010), explican que las iniciativas de trabajo vida se originaron en los años setenta en Estados Unidos y que en los años ochenta eran conocidas como políticas de trabajofamilia y estaban principalmente dedicadas a brindar facilidades en el cuidado de los niños de los empleados. Posteriormente, se comenzó a utilizar el término prácticas de trabajo-vida para indicar que todos los empleados necesitan apoyo para llevar una vida fuera del ámbito laboral.

A pesar de su amplia proliferación, las políticas que se diseñan para que los trabajadores integren el trabajo y la familia, no necesariamente reducen el conflicto trabajo-familia que el individuo experimenta (Kossek y Ozeki 1998). Fleetwood (2007) sostiene que algunos tipos de prácticas de balance trabajo-vida pueden favorecer este balance y otras pueden limitarlo, es decir que algunas pueden ser amigables para el empleado $y$ otras lo son para el empleador.

De acuerdo con Casey y Grzywacz (2008), tradicionalmente, la flexibilidad ha sido percibida como un recurso valorado por los trabajadores porquelespermitecoordinarsusresponsabilidades de trabajo con responsabilidades fuera del trabajo. La flexibilidad pone el control en manos de los trabajadores y hay evidencia substancial que sugiere que mayores niveles de control pueden ayudar a los trabajadores a responder significativamente a las demandas y responsabilidades de sus trabajos (Karasek y Theorell 1990, en Casey y Grzywacz 2008).

La flexibilidad laboral, fundamentalmente, se refiere a la habilidad de los trabajadores de modificar dónde, cuándo y cuánto tiempo se desarrolla el trabajo (Lewis 2003, en Casey y Grzywacz 2008). Hay dos formas dominantes de flexibilidad laboral: flexibilidad de horario y flexibilidad de ubicación.

Existen diversas prácticas de trabajo flexible (que son prácticas de conciliación vida personal-vida laboral) que muchas veces se denomina "atípico» o "formas no estandarizadas de empleo». Según como se le denomine en diferentes lugares puede cargar con una connotación positiva o negativa. A continuación se presenta un listado de diferentes tipos de trabajo flexible (O'Brien y Hayden 2008).

a) Horario flexible. Consiste en acudir al lugar de trabajo fuera de las horas establecidas como regulares. Normalmente se define cuáles son las horas en las que todos los trabajadores deben encontrarse en el centro de labores y se deja mayor flexibilidad para la entrada y la salida.

b) Trabajo a tiempo parcial. Consiste en que el empleado trabaje menos horas que las que constituyen una jornada a tiempo completo en la misma organización.

c) Trabajo compartido. Consiste en que un puesto de trabajo es compartido por más de una persona. Es muy positivo porque contribuye a que el trabajador disfrute de su estilo de vida y se mejoran aspectos como la retención del empleado y la posibilidad de contar con las habilidades requeridas en más de un trabajador. Por otro lado, requiere de gran comunicación entre las partes involucradas.

d) Teletrabajo. Es el trabajo que realiza una persona para su empleador desde una ubicación distinta a la tradicional utilizando las telecomunicaciones como elemento central del trabajo. El teletrabajo presenta como ventajas el ahorro de costos en espacios de oficina, el liberarse de congestiones de tráfico, el tener mayor autonomía, mejora el balance trabajo-familia, reduce la rotación y genera satisfacción laboral. Por otro lado, el teletrabajo tiene como 
desventajas el aislamiento del trabajador, la dependencia de la tecnología, el resentimiento de los colegas, la aparición de elementos distractores, la dificultad para evaluar el trabajo y el que no todos los trabajos sean apropiados para este tipo de esquema flexible.

e) Horarios anualizados. Permiten que el trabajador establezca sus patrones laborales para un año. Puede ayudar especialmente cuando se enfrenta estacionalidad en el negocio.

f) Horarios comprimidos. Son aquellos que comprimen semanas de trabajo, es decir que una jornada de 7 días se convierte en una de 3, 4 ó 4.5 días por semana, con más horas cada día. También se puede trabajar 10 horas diarias y de esa manera generar un día libre para actividades personales o en familia. Lo negativo es que el trabajador se sobrecarga durante los días en los que debe trabajar más horas y genera cansancio y estrés.

g) Años sabáticos. Se permite a los empleados dejar la organización por un año. Puede ser extendido a 2 o hasta 5 años.

h) Modelos de trabajo de por vida. Los períodos trabajados por sobre las jornadas normales se acumulan y utilizan a lo largo de la vida laboral.

i) Retiros anticipados. Una de sus modalidades consiste en permitir el retiro gradual del empleado combinando el entrenamiento de gente joven.

Según Lingard et al. (2007), las investigaciones indican que la introducción de iniciativas para conciliar la vida laboral-vida personal alivia el conflicto que los empleados experimentan entre trabajo y vida personal (Warren y Johnson 1995; VanRijswick et al. 2004, en Lingard et al. 2007) y facilita un mejor equilibrio entre el trabajo $y$ vida personal de los empleados (Tausig y Fenwick 2001; Madsen 2003 en Lingard et al. 2007). Muchas empresas han empezado a ofrecer sistemas de trabajo flexible para ayudar a los empleados a balancear sus demandas de trabajo y familia (Galinsky et al. 2008, en McNall et al. 2010). Las diferentes prácticas mencionadas pueden colaborar con que haya igualdad de oportunidades laborales para hombres y mujeres, pues en el caso de estas últimas se le ayuda a combinar las exigencias familiares que afronta.

\section{Metodología}

Se definió el modelo de regresión lineal simple para estimar el porcentaje de mujeres (del total que labora en la empresa) que ocupa cargos de nivel medio en empresas grandes a partir del número de prácticas de trabajo flexible que aplican las empresas empleadoras.

\section{Obtención de datos}

Los datos utilizados fueron obtenidos como parte de una encuesta aplicada en mayo de 2011, la cual aborda temas referentes al manejo sostenible de los recursos humanos en empresas peruanas (trabajo realizado con la Prof. Gina Pipoli de la Universidad del Pacífico). El cuestionario fue enviado a gerentes de recursos humanos de empresas grandes (que cuenten con más de 100 trabajadores) que operan en el Perú y que pertenecen a la Asociación Peruana de Recursos Humanos (Aperhu). Se eligió a las empresas grandes por ser ellas las que cuentan con prácticas de conciliación vida personal-vida laboral (sea por los presupuestos que manejan o por sus políticas internacionales). El cuestionario se realizó a través de un vínculo enviado en un correo electrónico. Se logró una respuesta del $40 \%$, recibiéndose 31 respuestas.

Se les preguntó a las empresas cuáles eran las prácticas de conciliación vida personal-vida laboral que utilizan y se procedió a cuantificarlas. Del mismo modo se les preguntó el porcentaje de mujeres que ocupa los cargos de nivel medio (es decir personal no operativo y personal no directivo).

El modelo

El modelo se sintetiza de la siguiente manera:

$\mathrm{Y}=\mathrm{Bo}+\mathrm{B} 1 \mathrm{X}$ 


\section{Donde:}

- La variable independiente (X) es el No de prácticas de trabajo flexible ofrecido por las empresas grandes. Se definió como una variable cuantitativa.

- La variable dependiente (Y) es el porcentaje de mujeres que ocupa cargos de nivel medio en las empresas grandes. Se definió como una variable cuantitativa.

Se utilizó el programa SPSS versión 19 para analizar los datos. Además de hallar los coeficientes de regresión, se comprobó que el modelo permite la estimación del porcentaje de mujeres que ocupan cargos de nivel medio en empresas grandes. Esto se hizo analizando el R2 y el estadístico F. Del mismo modo se procedió a verificar el nivel de significancia de los coeficientes del modelo. Cabe mencionar que se aplicaron todas las pruebas requeridas para comprobar que los supuestos necesarios para la regresión simple se cumplían. Estas pruebas se presentan a continuación.

\section{Revisión de supuestos de la regresión lineal simple}

A. La variable dependiente y la variable independiente son cuantitativas.

B. Homocedasticidad. Los residuales, a cada nivel de la variable predictora deben tener la misma varianza. Para realizar esta prueba se dividió a los residuos en dos grupos. G1 y G2. G1 para prácticas de conciliación de 0 a 5 y G2 para prácticas de conciliación de 6 a 9 . Como se puede apreciar en la tabla de resultados, el estadístico de Levene tiene una significancia de ,369 que es mayor que ,05 por lo que la homogeneidad de varianzas se puede asumir.

Prueba de homogeneidad de la varianza

\begin{tabular}{|c|c|c|c|c|c|}
\hline \multirow[b]{2}{*}{ Unstandardized } & \multirow[b]{2}{*}{ Basándose en la media } & $\begin{array}{l}\text { Estadístico } \\
\text { de Levene }\end{array}$ & gl1 & $\mathrm{gl} 2$ & Sig. \\
\hline & & ,831 & 1 & 29 & ,369 \\
\hline \multirow[t]{3}{*}{ Residual } & Basándose en la mediana & ,645 & 1 & 29 & ,429 \\
\hline & $\begin{array}{l}\text { Basándose en la } \\
\text { mediana y con } \\
\text { gl corregido }\end{array}$ & 645 & 1 & 28,590 & ,429 \\
\hline & $\begin{array}{l}\text { Basándose en } \\
\text { la media recortada }\end{array}$ & ,822 & 1 & 29 & ,372 \\
\hline
\end{tabular}

C. Independencia de errores. Se abordó la independencia de errores gráficamente y a través del estadístico Durbin-Watson. En ambos casos se comprobó la independencia de errores. El estadístico Durbin-Watson es 1,928. Es un valor cercano a 2 por lo que podemos asumir que los residuos no correlacionan. Valores menores que 1 o mayores que 3 serían causa de preocupación.

\section{Resumen del modelob}

\begin{tabular}{|c|c|c|c|c|c|}
\hline Modelo & $\mathrm{R}$ & $\mathrm{R}$ cuadrado & $\begin{array}{c}\text { R cuadrado } \\
\text { corregida }\end{array}$ & $\begin{array}{c}\text { Error típ. de la } \\
\text { estimación }\end{array}$ & Durbin-Watson \\
\hline 1 &, $850 \mathrm{a}$ &, 722 &, 713 &, 10850 & 1,928 \\
\hline
\end{tabular}

a. Variables predictoras: (Constante), No prácticas.

b. Variable dependiente: Mujcargomedio.

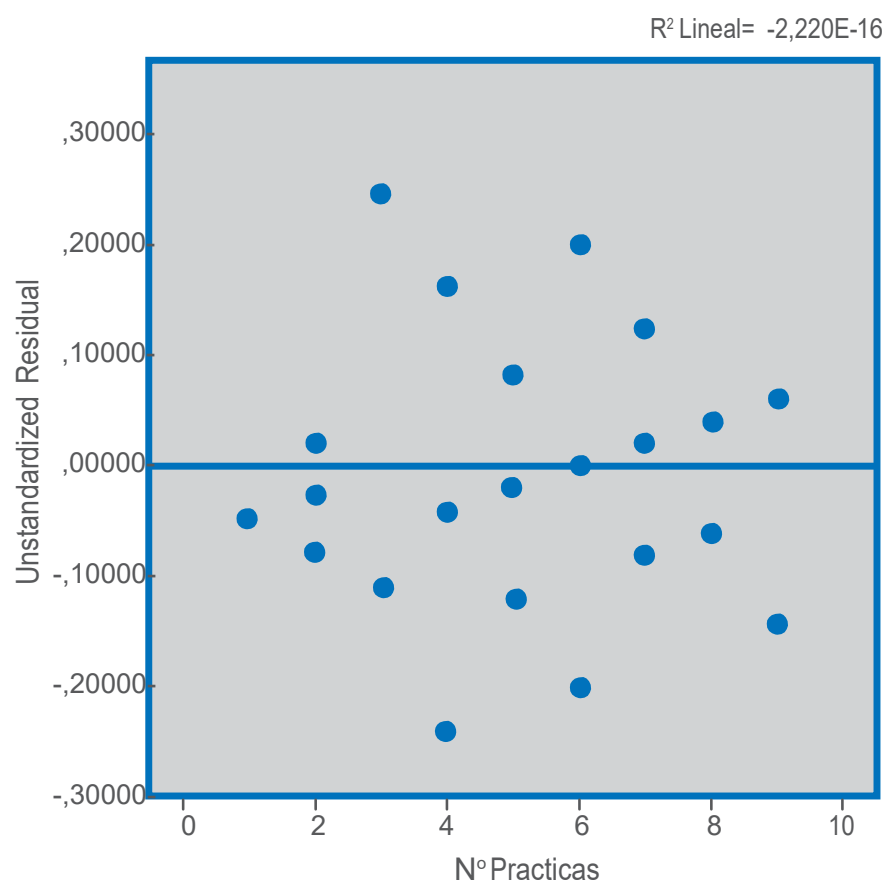

D. Distribución normal del error

Se realizaron varias pruebas.

a) Teorema del límite central

En muestras de 30 o más la distribución se aproxima a la normal.

b) P-P PLOTS e Histogramas

Gráficamente también se aprecia que los residuos se acercan a la normal. Los puntos se encuentran casi sobre la diagonal en el P-P PLOT y en el histograma también se aprecia la cercanía a la curva normal. 


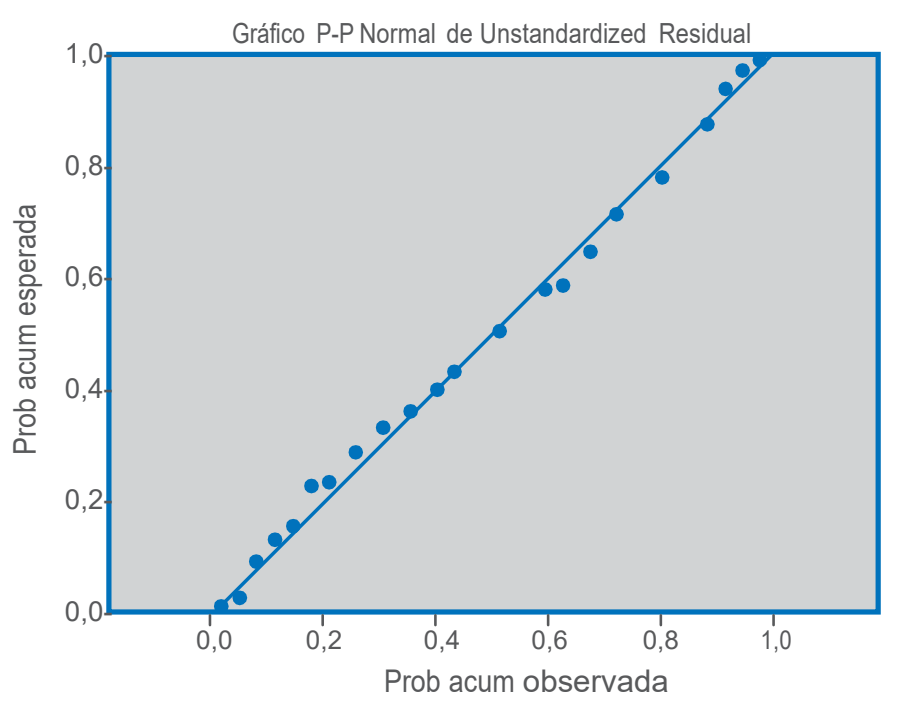

Media $=3,12 \mathrm{E}-17$ Desviación típica $=, 10667$ Histograma $\mathrm{N}=31$

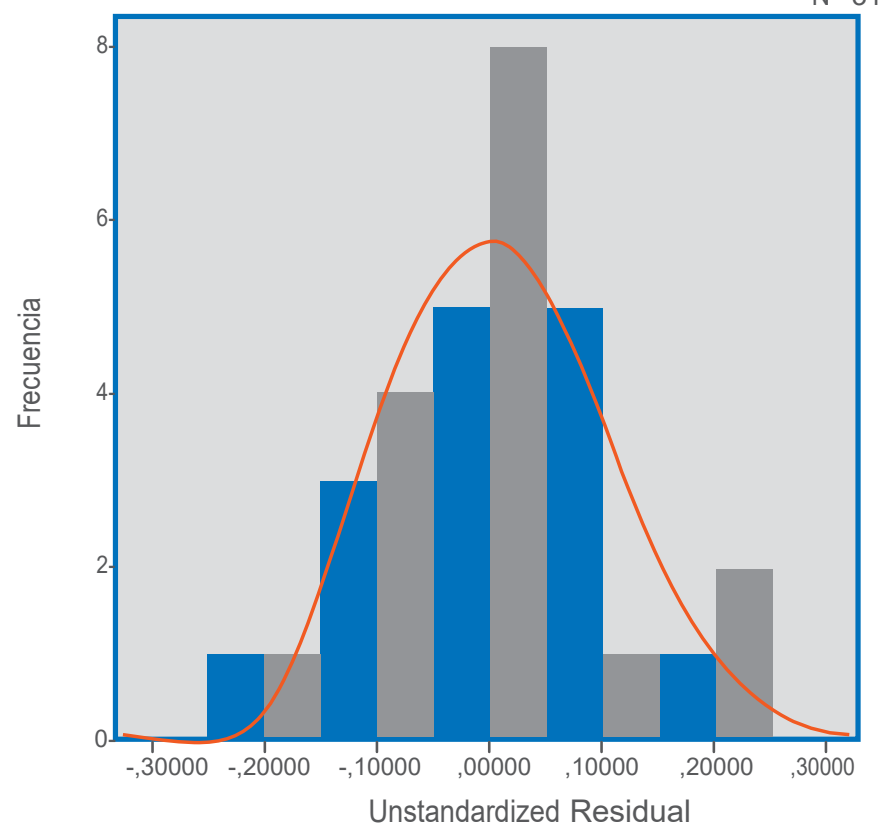

\section{c) Asimetría y curtosis}

La asimetría es, 074 . Al ser positiva indica que los valores positivos se acumulan a la izquierda y al ser muy cercana a cero, se aproxima a la normal. La curtosis es ,384. Al ser positiva indica una curva puntiaguda y con distribución cargada en las colas. A través de este análisis no se puede concluir que la distribución es normal porque la asimetría y curtosis no son cero pero se puede decir que tampoco se está tan alejado de la curva normal.

\section{Estadisticos}

\begin{tabular}{lc|r} 
Unstandardized Residual & $\begin{array}{c}\text { Válidos } \\
\text { Perdidos }\end{array}$ & 31 \\
Media & & 0 \\
Mediana & &, 0000000 \\
Moda &, 0012941 \\
Desv. típ. &, 00129 \\
Varianza &, 10667488 \\
Asimetría &, 011 \\
Error típ. de asimetría &, 074 \\
Curtosis &, 421 \\
Error típ. de curtosis &, 384 \\
Rango &, 821 \\
Mínimo & &, 48049 \\
Máximo & &,- 23772 \\
Suma & &, 24278 \\
Percentiles &, 00000 \\
& &,- 0596941 \\
& 25 &, 0012941 \\
& 50 &, 0817882
\end{tabular}

a. Existen varias modas. Se mostrará el menor de los valores.

\section{d) Kolmogorov-Smirnov Test y SahpiroWilk- Test}

Ambos tests son no significantes para un $p$ de ,05. Esto nos indica que la distribución de los residuales no es significativamente diferente de una distribución normal, así que es probablemente normal.

\section{Pruebas de normalidad}

\begin{tabular}{|l|c|c|c|c|c|c|}
\hline & \multicolumn{3}{|c|}{ Kolmogorov-Smirnov } & \multicolumn{3}{c|}{ Shapiro-Wilk } \\
\hline & Estadístico & gl & Sig. & Estadístico & gl & Sig. \\
\hline $\begin{array}{l}\text { Unstandardized } \\
\text { Residual }\end{array}$ &, 093 & 31 &, $200^{*}$ &, 987 & 31 &, 969 \\
\hline
\end{tabular}

a. Corrección de la significación de Lilliefors.

*. Este es un límite inferior de la significación verdadera.

En el gráfico $Q-Q$ también se aprecia que la distribución de los residuales se aproxima a la normal.

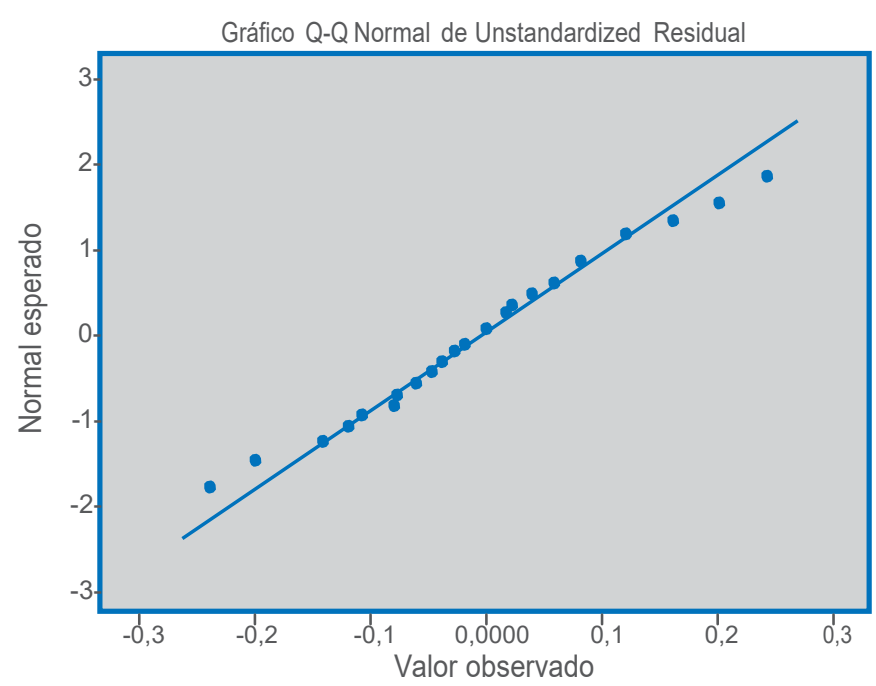




\section{Resultados}

El modelo de regresión da como resultado un $\mathrm{R} 2$ de ,722. Esto quiere decir que el número de prácticas de flexibilidad aplicadas por las empresas de la muestra, explica en un $72,2 \%$ la variación en el porcentaje de mujeres que ocupa cargos de nivel medio en estas empresas. Adicionalmente se puede apreciar que el estadístico $\mathrm{F}$ es 75,458 , lo cual indica que el modelo de regresión estima el porcentaje de mujeres que ocupa cargos de nivel medio significativamente bien.

Los coeficientes del modelo son $\mathrm{Bo}=, 016$ y $B 1=, 080$. El estadístico $t$, con su nivel de significancia de ,000 para una probabilidad de , 001 , indica que los valores de B1 son diferentes de cero y por lo tanto el número de prácticas de flexibilidad aplicadas por las empresas logra una contribución significativa para estimar el porcentaje de mujeres que ocupa cargos de nivel medio. La pendiente de la línea de ajuste total indica que a mayor número de prácticas de conciliación mayor será el porcentaje de mujeres en los cargos de nivel medio. A continuación se presenta el resumen del modelo.

\section{Resumen del modelo ${ }^{\mathrm{b}}$}

\begin{tabular}{|c|c|c|c|c|c|}
\hline Modelo & $\mathrm{R}$ & $\mathrm{R}$ cuadrado & $\begin{array}{c}\text { R cuadrado } \\
\text { corregida }\end{array}$ & $\begin{array}{c}\text { Error típ. de la } \\
\text { estimación }\end{array}$ & $\begin{array}{c}\text { Durbin- } \\
\text { Watson }\end{array}$ \\
\hline 1 &, $850 \mathrm{a}$ &, 722 &, 713 &, 10850 & 1,928 \\
\hline
\end{tabular}

a. Variables predictoras: (Constante), No prácticas.

b. Variable dependiente: Mujcargomedio.

ANOVA ${ }^{\text {b }}$
\begin{tabular}{|l|c|c|c|c|c|}
\hline Modelo & $\begin{array}{c}\text { Suma de } \\
\text { cuadrados }\end{array}$ & gl & $\begin{array}{c}\text { Media } \\
\text { cuadrática }\end{array}$ & $\mathrm{F}$ & Sig. \\
\hline $1 \quad$ Regresión &, 888 & 1 &, 888 & 75,458 &, $000^{\mathrm{a}}$ \\
$\quad$ Residual &, 341 & 29 &, 012 & & \\
\multicolumn{1}{|c|}{ Total } & 1,230 & 30 & & & \\
\hline
\end{tabular}

a. Variables predictoras: (Constante), No prácticas.

b. Variable dependiente: Mujcargomedio.

\section{Coeficientes $^{b}$}

\begin{tabular}{|l|c|c|c|c|c|}
\hline & \multicolumn{2}{|c|}{$\begin{array}{c}\text { Coeficientes no } \\
\text { estandarizados }\end{array}$} & $\begin{array}{c}\text { Coeficientes } \\
\text { tipificados }\end{array}$ & \multicolumn{2}{|c|}{} \\
\hline Modelo & B & Error típ. & Beta & t & Sig. \\
\hline 1 (Constante) &, 016 &, 053 & &, 295 &, 770 \\
№ prácticas &, 080 &, 009 &, 850 & 8,687 &, 000 \\
\hline
\end{tabular}

a. Variable dependiente: Mujcargomedio.
Cabe mencionar que las limitaciones del modelo son el tamaño de la muestra y la manera de aproximarse a la misma. Por limitaciones de tiempo el acercamiento a las empresas grandes se dio a través de los contactos que se tienen con APERHU (Asociación Peruana de Recursos Humanos) lo cual restringió la aplicación de las encuestas. Del mismo modo, al realizarse un cuestionario vía internet el encuestado ha podido incurrir en algún tipo de error o haber respondido la encuesta en un momento poco apropiado. Los resultados del estudio deberían validarse con una muestra mayor. Adicionalmente, al ser un modelo de regresión simple podrían estarse dejando de lado otras variables, como los años de nivel educativo o la capacidad de contratar servicio doméstico, para explicar el que la mujer tenga un mayor porcentaje de participación en la empresa. Es decir que, si bien las prácticas de conciliación vida personal-vida laboral parecen tener un efecto sobre la participación de la mujer en la empresa, pueden existir otras variables que también lo tengan y que no son abordadas en este estudio.

\section{Conclusiones}

Las prácticas de conciliación vida personal-vida laboral colaboran a que las mujeres puedan trabajar planteándose el desarrollo de su carrera a largo plazo. Las mujeres de nivel medio ya pudieron avanzar en su carrera y dejar el nivel operativo. El estudio realizado muestra que las prácticas de conciliación vida personal-vida laboral podrían explicar esta evolución en la carrera. Los resultados obtenidos pretenden despertar conciencia en el sector empresarial sobre lo beneficiosas que puede resultar la aplicación de este tipo de prácticas, especialmente en un entorno en el cual el talento se quiere retener. Vías futuras de investigación deberían acercarse a la realidad de la mujer en el nivel directivo puesto que es allí donde mayores dificultades tiene la mujer para permanecer. Es la igualdad de oportunidades laborales para hombres y mujeres lo que realmente se busca y las prácticas de conciliación vida personal-vida laboral pueden contribuir en ese objetivo. 


\section{BIBLIOGRAFÍA}

BARSH, J. y L. Yee

2011 Changing companies' minds about women. McKinsey Quarterly.

BROWN, L.

2010 The relationship between motherhood and professional advancement. Employee Relations, 32 (5), 470-94.

CASEY, P. y J. Grzywacz

2008 Employee Health and Well-Being: The role of flexibility and work-family balance. The Psychologist - Manager Journal, 11, 31-47.

CATALYST

2009 Women Leaving and Re-entering the Workforce. Recuperado el 11 de junio de 2010, de Catalyst: http://www.catalyst.org/page/64/ browse-research-knowledge.

CHANG, Artemis, McDonald, P. y Burton, P.

2010 Methodological choices in work-life balance research 1987-2006: a critical review. The International Journal of Human Resource Management, 21(13), 2381-2413.

\section{DBM PERÚ}

2009 DBM. Recuperado el 12 de junio de 2010, de http://www.dbmperu.com/pdf-estudiosmundiales/genero.pdf.

EDWARDS, J. R. y N. P. Rothbard

2000 Mechanisms linking work and family: clarifying the relationship between work and family constructs. Academy of Management Review , 25 (1), 178-99.

FIELD, Andy.

2009 Discovering Statistics using SPSS. SAGE Publications. Third edition.

FLEETWOOD, S.

2007 Why work-life balance now? International Journal of Human Resource Management, 18(3), 387-400.

\section{FORTUNE}

2010 CNN Money. 3 de mayo de 2010, recuperado el 13 de agosto de 2010, de http://money. cnn.com/magazines/fortune/fortune500/2010/ womenceos/.

GARCÍA, M.

2010 Tierra de Hombres. América Economía , 52-3.
GRZYWACZ, J. G. y D. S. Carlson

2007 Conceptualizing Work-Family Balance: Implications for Practice and Research. Advances in Developing Human Resources, 9(4), 455-71.

LINGARD, H., K. Brown, L. Bradley, C. Bailey, y K. Townsend

2007 Improving Employees' Work-Life Balance in the Construction Industry: Project Alliance Case Study. Journal of Construction Engineeringand Management, 807-15.

ILO

2010 Women in labor markets: Measuring progress and identifying challenges.

\section{INSTITUTO NACIONAL DE ESTADÍSTICA EN INFORMÁTICA (INEI)}

2011 Encuesta Nacional de Uso del Tiempo 2010. Lima: Instituto Nacional de Esatadística e Informática.

KOSSEK, E. E., S. Lewis, y L. Hammer

2010 Work-life initiatives and organizational change: overcoming mixed messages to move from the margin to the mainstream. Human Relations, 63(1), 3-19.

KOSSEK, E. E., y C. Ozeki

1998 Work-family conflict, policies, and the job-life satisfaction relationship: a review and directions for organizational behavior-human resources research. ournal of Applied Psychology, 83(2), $139-49$.

LEWIS, S., R. Gambles y R. Rapoport

2007 The constraints of a «work-life balance» approach: an international perspective. International Journal of Human Resource Management, 360-73.

O'BRIEN, T., y H. Hayden

2008 Flexible work practices and the LIS sector: balancing the needs of work and life? Library Management, 29 (3), 199-228.

McNALL, L. A. Masuda y J. Nicklin

2010 Flexible Work Arrangements, Job Satisfaction, and Turnover Intentions: The Mediating Role of Work-to-Family Enrichment. The Journal of Psychology, 144 (1), 61-81.

MINISTERIO DE TRABAJO Y PROMOCIÓN DE EMPLEO (MINTRA)

2009 Ministerio de Trabajo y de Promoción del Empleo. 27 de mayo de 2009, recuperado en 
setiembre de 2010, de http://www.mintra.gob. pe/archivos/file/estadisticas/peel/estadisticas/ cuadro95.pdf.

POWELL, G. N., y J. H. Greenhaus

2010 Sex, gender, and the work to family interface: exploring negative and positive interdependencies. Academy of Management Journal , 53 (3), 513-34.

RANSOME, $P$.

2007 Conceptualizing boundaries between «life» and «work». International Journal of Human resource Management, 374-86.

RYAN, A. M., y E. E. Kossek

2008 Work-life policy implementation: breaking down or creating barriers to inclusiveness? Human Resource Management, 47(2), 295310.
STURGES, J

2008 All in a day's work? Career self-management and the management of the boundary between work and non work. Human Resource Management Journal, 18(2), 118-34.

STURGES, J. y D. Guest

2004 Working to live or living to work? Work/life balance early in the career. Human Resource Management Journal, 14(4), 5-20.

\section{WORLD ECONOMIC FORUM}

2010 The Global Gender Gap Report. Recuperado en noviembre de 2010, de http://www. weforum.org/en/Communities/Women $\% 20$ Leaders\%20and\%20Gender\%20Parity/ GenderGapNetwork/index.htm. 